Slavica

bruxellensia

\section{Slavica bruxellensia}

Revue polyphonique de littérature, culture et histoire

slaves

4 | 2009

Espaces slaves / espaces belges

\title{
Michel de Ghelderode en République tchèque
}

\section{Honza Petružela}

Traducteur : Sarah Flock

\section{(2) OpenEdition}

Journals

Édition électronique

URL : http://journals.openedition.org/slavica/262

DOI : $10.4000 /$ slavica.262

ISSN : 2034-6395

Éditeur

Université libre de Bruxelles - ULB

Édition imprimée

Pagination : 22-33

ISSN : 2031-7654

Référence électronique

Honza Petružela, "Michel de Ghelderode en République tchèque », Slavica bruxellensia [En ligne],

4 | 2009, mis en ligne le 15 octobre 2009, consulté le 01 mai 2019. URL : http://

journals.openedition.org/slavica/262 ; DOI : 10.4000/slavica.262

Ce document a été généré automatiquement le 1 mai 2019.

Les contenus de Slavica bruxellensia sont mis à disposition selon les termes de la Licence Creative Commons Attribution - Pas d'Utilisation Commerciale - Pas de Modification 3.0 France. 


\title{
Michel de Ghelderode en République tchèque
}

\author{
Honza Petružela \\ Traduction : Sarah Flock
}

\section{NOTE DE L'ÉDITEUR}

La langue originelle de cet article est le tchèque

1 Les articles scientifiques qui, dans une culture théâtrale nationale, recensent la tradition scénique des pièces étrangères traduites se consacrent souvent aux auteurs classiques et confirmés. Pour ces auteurs, on suppose souvent que l'analyse des mises en scène nous rapproche de la perception de l'œuvre à une époque donnée et enrichit notre connaissance de cette même époque. En allant plus loin, on peut aussi déceler quelque chose sur la culture théâtrale étudiée, même s'il reste évident qu'elle se laisse alors facilement coincer entre une généralisation séduisante et le mythe théâtral «national ", surtout si le travail ne se base pas sur une comparaison transnationale élargie du phénomène donné. Pour les auteurs rejetés à la périphérie de l'intérêt dramaturgique et en dehors des courants principaux, la situation, apparemment encore plus compliquée, se caractérise principalement par un manque de sources, même si les bribes d'informations collectées peuvent toutefois introduire de nouveaux éléments dans les rapports théâtraux et culturels, et les éclairer d'une lumière inattendue. Le dramaturge belge Michel de Ghelderode (1898-1962) est l'un de ces auteurs.

2 La majorité des études qui s'intéresse à la dramaturgie de Ghelderode oscille entre deux pôles. Certaines constatent que Ghelderode est peut-être l'auteur belge le plus joué en Europe alors que d'autres déplorent que l'œuvre scénique du "Shakespeare flamand " n'est pas assez jouée. La réflexion sur la réception de Ghelderode porte sur ses textes mêmes, indépendants et originaux, mais aussi, pour beaucoup, insaisissables et, d'un point de vue théâtral, incompréhensibles. La littérature scientifique recense quelques 
études sur le thème de la réception théatrale de ces textes ${ }^{1}$. Dans le présent article, nous tenterons d'esquisser tout au moins les gros traits de la tradition des traductions et mises en scène des pièces de cet auteur dans le contexte théâtral de la République tchèque et ainsi de contribuer peut-être à la continuation de cette oscillation ambiguë.

Les pièces de Ghelderode jouirent internationalement de plusieurs vagues d'intérêt. La première, celle qui le révéla au monde, fut qualifiée de "ghelderodite aiguë $\rrbracket^{2}$ dans les théâtres parisiens à la fin des années 1940 et au début des années 1950. Ces premières vagues qui trouvaient un écho dans la dramaturgie occidentale se brisèrent néanmoins à l'Est sur le «Rideau de fer ». Le développement d'après-guerre dans la Tchécoslovaquie du début des années 1950 évitait les contacts quels qu'ils furent du théâtre tchèque avec l'Occident "impérialiste ", ce qui empêcha les auteurs occidentaux non tolérés de percer sur les scènes tchèques et rendaient les traductions de la littérature occidentale généralement inaccessibles. Il n'est pas inutile de rappeler qu'à l'époque du plus vif intérêt mondial pour Ghelderode, la Tchécoslovaquie vivait au rythme des procès politiques orchestrés par les commissaires soviétiques qui se soldèrent par de nombreuses condamnations à mort. En tant qu'auteur de farces " macabres », Ghelderode aurait certainement incarné pour les autorités communistes une forme d'entartete Kunst occidental et, par conséquent, n'aurait pu, même hypothétiquement, intégrer la ligne du parti qui régissait le système théâtral.

4 En 1957, les Polonais furent les premiers à briser l'interdiction de traduire et de monter Ghelderode en Europe centrale et orientale ${ }^{3}$. En 1964, la presse russe mentionna l'auteur pour la première fois, il fallut toutefois attendre l'année 1975 avant que ne parût une première traduction en russe. Et il fallut attendre jusqu'en 1978 avant que ne fût éditée une traduction hongroise $e^{4}$. A la fin des années 1950, bien qu'il y eût toujours des prisonniers politiques, la Tchécoslovaquie connut une période de relâchement partiel du terrible régime stalinien. C'est ainsi que l'on put trouver des titres jusqu'alors impensables d'auteurs étrangers dans la dramaturgie théâtrale tchèque. En 1964, les premières de En attendant Godot de Samuel Beckett et de La Cantatrice chauve d'Eugène Ionesco eurent lieu au Divadlo na zábradlí (Théâtre sur la balustrade).Au début des années 1960, les premières pièces de Václav Havel y firent également leur apparition. Il est alors symptomatique que la première traduction et la première représentation de Ghelderode n'advinrent qu'en cette période de dégel.

En 1965, le célèbre metteur en scène Otomar Krejča fonda le désormais légendaire Divadlo za branou (Théâtre derrière la porte). La personnalité de Krejča, l'une des plus remarquables de la mise en scène tchèque, se dégagea considérablement à la charnière des années 1950 et 1960, lorsqu'il créa conjointement avec son dramaturge Karel Kraus une ère significative pour le Národní divadlo (Théâtre national) avec la mise en place d'un atelier dramaturgique pour auteurs. Kraus est également le père de la première traduction de la pièce de Ghelderode en République tchèque. Masques ostendais, sketch pantomime pour le moins étonnant, qui fut joué le 23 novembre 1965 à l'occasion de l'inauguration du théâtre, fut suivi par l'œuvre dramatique Kočka na kolejích(La Chatte sur les rails) de Josef Topol. Ainsi, ce fut par une pièce belge que débuta l'activité glorieuse de la remarquable troupe du théatre za branou qui connut une fin si agitée ${ }^{5}$. Cette courte farce pantomime à l'atmosphère carnavalesque fut mise en scène avec de grands masques réalisés par le plasticien renommé Jan Koblasa ${ }^{6}$ et liée au drame poétique de Topol sur la relation entre deux êtres. Ce drame exprimait les sentiments de sa génération et constitua un contrepoint appuyé dans le programme de la soirée. Le monde dansant des 
masques cruels où s'interpénétraient le rêve et la réalité (et qui évoquait aussi la célèbre pièce de Topol Konec masopustu[Fin de Carnaval], dans laquelle les masques jouèrent un rôle important) ouvrait la soirée, et annonçait le drame entre un homme et une femme dans une station de train perdue au milieu de la nuit, un drame intime mais d'autant plus percutant et poétique. Masques ostendais présentait le contexte au plus haut point théâtral de La chatte sur les rails, un drame basé sur le texte. La critique de l'époque encensa fortement ce bref acte inaugural ; elle remarqua surtout l'aspect esthétique inspiré des masques de James Ensor, la conception non traditionnelle de la plastification pantomimique ainsi que la danse colorée et rythmique qui ne rendaient pas l'interprétation évidente pour le spectateur. Les deux actes étaient également liés entre eux à l'aide de motifs musicaux et de détails scéniques (motif des masques et des visages cachés). Les deux représentations, ayant connu en tout cent cinquante-cinq reprises, furentmême jouées en Belgique lors d'une tournée en 19697. L'arrivée de Ghelderode sur la scène tchèque fut donc liée, dans la mise en scène de Krejča, à l'activité intensive du théâtre-atelier nouvellement créé dont la recherche portait sur la forme scénique, sur le développement dramaturgique et sur celui des acteurs. Afin d'esquisser un paysage complet du contexte de l'époque, rappelons encore qu'en 1965, parut un numéro thématique de la revue spécialisée «DIVADLO », dédié à Antonin Artaud".

6 La primauté des traductions et mises en scène de Ghelderode revint incontestablement au Divadlo za Branou de Krejča. Cependant, un plus vaste public allait assimiler le nom de Ghelderode aux premières traductions et études de Jiří Konůpek. Sans la publication de ses dix excellentes traductions, la découverte de l'œuvre de Ghelderode en République tchèque aurait vraisemblablement été fortement retardée, comme en témoignent les exemples susmentionnés de la Russie ou de la Hongrie. En 1966, la présentation des Masques ostendais, les revues « DIVADLO » et« SVĚTOVÁ LITERATURA », qui proposaient alors en Tchécoslovaquie de très bonnes traductions d'œuvres internationales ne pouvant paraître sous forme de livre, publièrent trois courts drames : Trois acteurs, un drame ${ }^{9}$, Les Aveugles ${ }^{10}$ et Christophe Colomb ${ }^{11}$. La traduction d'une des pièces fondamentales de l'œuvre de Ghelderode, La Balade du Grand Macabre ${ }^{12}$ sortit aux éditions de l'agence littéraire Dilia qui était alors principalement accessible aux personnes de théâtre. L'année suivante parut $L a$ pie sur le gibet - Pantagleize ${ }^{13}$. L'action littéraire la plus essentielle (et la seule véritable pour de longues années) survint également en 1966 lorsque la maison d'édition Orbis (au sein de laquelle, grâce à ses rédacteurs érudits, paraissait de nombreux titres de la littérature internationale) publia, un recueil intitulé Magie rouge et autres pièces ${ }^{14}$ qui regroupait quatre textes: Hop, Signor!, Magie rouge, D'un diable qui prêcha merveilles et Sire Halewyn. Aussi, si l'on inclut la traduction non publiée des Masques ostendais la Tchécoslovaquie comptait-elle, en 1967, onze traductions de pièces de Ghelderode.

7 Si on omet le bref encart biographique figurant sur le programme de la mise en scène de Krejča en 1965, qui finalement constituait la première mention de Ghelderode en République tchèque, les premiers textes fondamentaux sur l'œuvre et la vie de l'auteur n'arrivèrent qu'après les publications des traductions. Leur auteur est à nouveau Konůpek qui, en un an, publia trois articles sur ce sujet. Le premier était plutôt un article biographique, «Tajůplný Michel de Ghelderode »" (Le mystérieux Michel de Ghelderode) paru dans « SVĚTOVÁ LITERATURA », puis un second plus essentiel, « Tanec života a smrti » (Danse de la vie et de la mort) publié dans la revue "DIVADLO", article dans lequel il relevait entre autres la filiation avec le théâtre de la cruauté d'Artaud, plaçait l'oeuvre de Ghelderode dans le contexte des idées de l'époque, en refusait une interprétation 
unilatérale et sombre et faisait observer la danse contradictoire du bien et du mal, de la vie et de la mort. Enfin, dans son recueil de pièces admirablement conçu, parut sa postface intitulée Dramatické dilo Michela de Ghelderode (L'œuvre dramatique de Michel de Ghelderode) ${ }^{16}$. Malheureusement, Konůpek se base ici sur un livre d'Albert Lepage ${ }^{17}$ influencés par les mystifications de Ghelderode et qui est donc chargé d'une série d'inventions imaginées par l'auteur. Il faut évidemment mettre ce fait sur le compte de l'inaccessibilité effective d'autres sources pertinentes à l'époque ; néanmoins les erreurs citées, principalement concernant la datation de la plupart des titres, furent transmises dans d'autres textes courts publiés par la suite. En 1968, à nouveau dans la revue " DIVADLO ", Jindřich Černý remarqua l'œuvre fondatrice de Konůpek lorsqu'il recensa en détail les traductions et surtout les interpréta en fonction du contexte de l'époque. Pendant ces temps houleux de rénovation du socialisme et de tentative pour instaurer un socialisme à visage humain, temps plus connu sous le nom du "Printemps de Prague " auquel mit fin l'invasion soviétique d'août 1968, son interprétation de Christophe Colomb résonnait éloquemment :

Colomb se serait-il échappé dans les régions libres de l'illusion, aurait-il vogué jusqu'au diable s'il avait vécu dans son royaume? J'ai bien peur que non. La souffrance de Colomb découle justement du fait qu'il ait vécu dans un royaume d'Ordre qui, bien qu'il fût peut-être une œuvre divine, s'était pourtant retrouvé entre les mains de fieffés idiots ${ }^{18}$.

8 Exceptions faites de certaines préférences nationales pour des titres particuliers ${ }^{19}$, il est typique dans la dramaturgie de Ghelderode d'introduire ses œuvres par vagues qui, comme nous l'avons abordé plus haut, après un gonflement tempétueux retombent vite. La situation tchèque a la particularité d'être liée au développement historique des années proches de 1968 dont le processus régénérateur fut jugulé par l'invasion des armées "amies» du Pacte de Varsovie sur le territoire tchécoslovaque (et leurs séjours d'occupation provisoires), suivie par vingt ans de «normalisation » complète. Le texte de Černý, qui allait devenir plus tard le directeur du théâtre national, retentit comme un appel direct et concret aux professionnels de la scène : « Il est plus que temps maintenant que nous prenions connaissance de l'oeuvre de Ghelderode et que nous ayons sur elle un point de vue personnel-que nous n'attendions pas que seuls des dramaturges tchécoslovaques hésitants le fassent. $»^{20}$ Concrètement, la première vague d'intérêt pour Ghelderode sur la scène tchèque et certainement la plus remarquable jusqu'à nos jours se déroula de 1969 à 1973, lorsqu'il était encore possible de décliner, dans une certaine mesure, un répertoire non conformiste et de produire un nouveau théâtre d'inspiration, bien que la normalisation n'eût déjà clairement indiqué les démarches percutantes de son avènement (le Divadlo za Branou de Krejča fut supprimé en 1972, par exemple). Pendant cette période, allaient être créées cinq mises en scène de Ghelderode d'après les traductions de Konůpek. Après leurs publications, les textes du traducteur, qui décéda malheureusement en 1968, furent rapidement saisis par de jeunes metteurs en scène alors débutants qui allaient ensuite œuvrer au sein d'ensembles alternatifs de renom et dont certains allaient devenir plus tard des légendes du théâtre tchèque.

9 Dans un nouveau théâtre de Brno, le Divadlo Husa na provázku (Théâtre l'Oie en laisse), qui allait devenir le représentant le plus marquant du mouvement des studios de théâtre à l'instar du Théâtre de Haná (Hanácké divadlo), du Studio Y ou du Studio dramatique ( Činoherní studio), Eva Tálská mit en scène le sombre Sire Halewyn au printemps $1969^{21}$. La dramaturgie ouverte, qui comprend également un choix de textes de Ghelderode,était l'une des caractéristiques des studios de théâtre. Metteur en scène à l'imaginaire poétique, 
ayant un goût pour l'expérimentation dramaturgique et la métaphore scénique, Tálská dont l'une des premières réalisations fut, par exemple, l'adaptation théâtrale de l'œuvre poétique Les chansons du gibet de Christian Morgenstern, aborda précisément la pièce de Ghelderode dans le même esprit. Mettant en scène la cruelle essence des passions humaines, son Sire Halewyn se tenait parmi les spectateurs dans un espace vide sans accessoires. L'allocution des acteurs, stylisée de manière expressive, se rapprochait du théâtre de mouvement.Chaque personnage avait un geste rythmique spécifique, qui un mouvement, qui un son (marche à reculons, à quatre pattes, martèlement des paumes sur le sol...). D'après la metteur en scène, «le corps même des acteurs était comme cousu par un son » etla stylisation affranchie accentuait la passion des personnages exaltés à la limite même de l'humanité.Les acteurs portaient des costumes noirs homogènes (jeans et pull-overs) et leurs partenaires étaient surtout le chœur qui incarnait, par exemple, le miroir de Halewyn et complétait l'atmosphère (utilisation de modèles rythmiques, de rassemblements de sons...). Cette mise en scène s'inscrivit dans le contexte de l'époque manifestement très inspirant, celui du théâtre de la cruauté d'Artaud, des expériences de Jerzy Grotowski et de la poétique du Living theatre, qui, dans l'union entre Tálská et Ghelderode, trouva un épanouissement personnalisé et, dès le début, l'une des représentations les plus originales de l'auteur en République tchèque.

En cette même ville, sur le sol de l'académie de théâtre (JAMU), se tint la même année une mise en scène de Trois acteurs, un drame... ${ }^{22}$, pièce jouée par des étudiants et dont les entractes reprenaient des airs forains. Mais la mise en scène phare devait seulement arriver. En 1969, Karel Makonj, fraîchement diplômé du département de mise en scène de marionnettes de la DAMU, fonda à Prague avec ses contemporains le Vedené divadlo (Théâtre dirigé). Comme l'insinuait déjà son nom, il s'agissait d'un théâtre de marionnettes et d'acteurs, pas d'un théâtre de marionnettes selon l'acceptation courante du terme, mais bien d'un ensemble expérimental influencé par les tendances de laboratoire développées dans les années 1960. L'orientation de son programme, sa réflexion théorique sur les phénomènes de la marionnette et de la matière, sa dramaturgie bien fondée et ses interprétations étonnantes de la mise en scène rendirent, selon moi, ce théâtre unique même sur la scène européenne de l'époque. Le Vedené divadlo , théâtre ambulant qui ne possédait pas de salle permanente à Prague, s'adressait à sa génération et ce, dès sa première mise en scène, Le Malentendu d'Albert Camus ${ }^{23}$, dans laquelle jouaient tant des acteurs de chair que des marionnettes en bois de stère dotées d'un mécanisme articulaire développé qui doublaient les personnages de la pièce. Le jeu d'ensemble ou plutôt le contre-jeu des acteurs et marionnettes incarnait le principe de la mise en scène, soit la représentation de rapports complexes entre les personnages ou à l'intérieur de soi-même. Si Makonj observait dans cette pièce la détermination humaine du côté intérieur, dans les intentions suggérées par la philosophie camusienne, le choix de Hop, Signor $!^{24}$ en 1970 déplaça plus profondément sa recherche expérimentale vers les mystères de l'essence humaine, vers la détermination intérieure.

11 Les personnages de Hop, signor! n'ont pas une nature psychologique complexe, polysémique ou simple. Ils sont consumés par leurs passions exacerbées à l'extrême. On pourrait affirmer que les caractères de cette farce carnavalesque tragique sont littéralement guidés et entrainéspar leurs passions. À partir de ce constat, il n'y a plus qu'un pas à franchir pour atteindre le principe de mise en scène qu'utilise Makonj : ce dernier allait nouer des fils rouges non pas aux marionnettes mais bien aux comédiens eux-mêmes et les laisser être menés à travers la scène par des personnages énigmatiques 
perchés en haut d'un banc en bois déformé et vêtus de blouses de chirurgien dont les silhouettes se perdaient dans l'obscurité. Il ne s'agit pas ici de la métaphore classique du grand marionnettiste, mais d'un principe bien plus sophistiqué, celui de l'homme dépourvu des forces qu'il aurait pu maîtriser, celui des cordes qui se tendent et se raidissent car elles sont manipulées par les instincts. Cela n'a rien à voir avec la marionettisation passive des personnages de Maeterlinck ni avec la détermination des personnages de pièces absurdes ensablés jusqu'au cou, mais bien avec la liberté absolue de corps et d'esprit qui revient en hémicycle, pour reprendre la terminologie de Rio Preisner ${ }^{25}$, vers le point de détermination absolue. Le thème de l'aliénation du sujet dans cette dialectique affreuse de liberté et d'assujettissement ne doit ni s'expliquer politiquement, ni d'après ce que pensaient les créateurs eux-mêmes. Toutefois, par son choix dramaturgique de textes "sombres" et existentiels, le Vedené divadlo faisait intérieurement et tout à fait clairement écho à l'atmosphère du malaise pourrissant d'après 1968.

Dans sa recherche du texte adéquat qui authentifierait son principe de mise en scène, dans ses efforts pour dévoiler l'intérieur par le biais de l'extérieur, Makonj comprit (je le souligne) de manière précise les qualités intrinsèquement théâtrales des écrits de Ghelderode qui sont pourtant trop souvent classés dans la catégorie "littérature » et pour lesquels on ne va souvent pas plus loin qu'une illustration grotesque des mots et des phrases qu'ils contiennent. Bien qu'il ne s'agitt « seulement » que d'une expérience qui ne réussit pas totalement, on peut vraiment parler selon moi de l'interprétation tchèque la plus originale de Ghelderode. Afin d'expliquer l'intérêt surprenant des théâtres parisiens pour l'auteur à la fin des années 1940 , un critique de l'époque parmi d'autres affirma: "C'est la mode, voilà tout. [...] Paris éprouve périodiquement le besoin de respirer ainsi les brouillards du Nord. Maeterlinck, Crommelynck, Ghelderode... $»^{26}$ Toutefois, Roland Beyen désigne surtout l'opposition au théâtre philosophique de Camus et de Jean-Paul Sartre comme l'une des raisons de l'apparition de Ghelderode sur les scènes parisiennes dans les années 1940. Aussi, au-delà du contexte tchèque, il est intéressant que le metteur en scène Makonj ait placé, à la charnière des années 1960 et 1970, Camus et Ghelderode côte à côte dans le programme dramaturgique du Vedené divadlo. Son interprétation liait Le Malentendu à Hop, Signor!,drames sur la liberté et sur l'humanité rendus de manière originale par les liens entre les marionnettes et les comédiens.

En 1970, à Ostrava, le récit cruel de la farce Hop, Signor ! figurait également au programme du Divadlo Petra Bezruče (Théâtre Petr Bezruč). À en croire les critiques de l'époque, la pièce, dans laquelle étaient utilisés de grands masques céphaliques et où la voix des acteurs était enregistrée sur des bandes magnétiques, ne connut pas un vif succès. L'année suivante, à Brno, le marionnettiste Pavel Vašíček mit en scène une adaptation très personnelle de la courte farce Les aveugles ${ }^{27}$. Le metteur en scène se basait ici sur le principe du "théâtre dans le théâtre » alors que des comédiens de la «Bande du jugement dernier ", menée par le personnage de Lord Macabre, incarnant lui-même par Lamprido, manipulaient des marionnettes. Sur scène, il y avait un groupe de carnavaliers qui représentait des personnes grotesquement stylisées pour lesquelles toute la pièce était jouée comme si elle détenait un message et un avertissement. L'adaptation textuelle, quant à elle, reprenait des motifs d'autres pièces de Ghelderode et ainsi mettait vraisemblablement à jour une des lectures les plus marquantes de Ghelderode en République tchèque. En 1973, Jan Schmid, metteur en scène du Studio Ypsilon à Liberec, clôtura la saison par la Balade du Grand macabre ${ }^{28}$. Avec une conclusion étrange et 
faussement optimiste de la pièce apocalyptique où les amants sortent de leurs tombes pour rencontrer une vie nouvelle et où le Prince Goulave nomme ministres du nouveau gouvernement les ivrognes Porprenaz et Videbolle, l'œuvre "sombre» de Ghelderode disparut comme par enchantement ce qui est très caractéristique des treize années de normalisation qui allaient suivre.

La découverte des œuvres de Ghelderode grâce aux mises en scène d'artistes célèbres tels que Krejča, Tálská, Makonj, Vašíček et Schmid dut cesser pendant treize ans, de 1973 à 1986. Makonj allait à nouveau rompre ce silence en montant la pièce Balade du Grand macabre sous le titre de Nekrozotar, sans utiliser cette fois-ci de marionnettes mais en présentant son adaptation en plein air. La représentation se tint dans le cadre de la Divadelní pou ̌̌ (Kermesse théâtrale) qui était un petit festival estival pragois auquel prenaient part des créateurs renommés issus du théâtre alternatif et de studio ${ }^{29}$. En 1986, Schmid revint également vers Ghelderode en présentant la pièce satyrique D'un Diable qui prêcha merveilles ${ }^{30}$. Le texte, initialement écrit, comme nous le savons, pour des marionnettes, fut joué par des acteurs sans marionnettes, totalement dans l'esprit de la poétique originale du Studio Ypsilon: la structure de la pièce fut retouchée, de nouvelles situations et des chansons furent ajoutées, les comédiens brisèrent l'illusion théâtrale, incorporèrent des commentaires personnels sur le thème de la bestialité et de la bêtise humaines. Ces deux retours aux textes de Ghelderode de la part de metteurs en scène confirmés indiquaient un choix dramaturgique réfléchi qui répondait à une poétique propre et à une adoption des thèmes. Chez Makonj, il s'agissait du grotesque ainsi que de la parabole de la manipulation et du sort métaphysique de l'homme que proposaient les marionnettes; pour Schmid il s'agit de la coquetterie d'un genre léger, du jeu d'acteur basé sur l'improvisation et d'une farce crue. Avec un brin d'exagération, nous pourrions peut-être déceler dans les titres des deux pièces présentées la fin approchante du gouvernement des macabres et des diables normalisés qui se promettaient des merveilles surtout dans le contexte du régime communiste périclitant.

Avec la Révolution de Velours de novembre 1989 prit fin quarante années de pouvoir totalitaire; avec la chute du Rideau de fer, s'écroula aussi toute restriction de censure (bien que la censure n'existait officiellement plus depuis les années 1960) et la culture théâtrale libre s'ouvrit au monde dramatique tout entier. À Ostrava, en mars 1990, la troupe du Divadlo Petra Bezruče ajoutait déjà à son répertoire le drame Barabas, jusqu'alors non monté. Pour les costumes des acteurs, le metteur en scène choisit quelques signes relevant de la convention des cabarets et du music hall. Il se rapprochait ainsi de la forme du grotesque qui maintenait surtout l'alternance de la tension entre la description et l'allusion scénique. Du point de vue contemporain, le choix d'une pièce renversant un épisode de la Passion, comme nous en trouvons plusieurs chez Ghelderode ${ }^{31}$, évoque de manière quelque peu effrayante une préfiguration spontanée de l'évolution de la République tchèque libre, car Barabas est un drame très amer et porteur d'une vision apocalyptique sans morale où dirigent des bouffons et des Barnum. Que l'on daigne se rappeler les fraudes et le socialisme bancaire, la dilapidation des biens de l'État par de nouveaux entrepreneurs rapidement apparus et par de nouvelles structures ayant enfilé de nouvelles vestes dans la première moitié des années 1990 ainsi que certaines méthodes du marasme éthique qui perdure encore aujourd'hui. Mais arrêtons ici avec ces analogies acerbes.

Dans les années 1970 et 1980, l'absence de mise en scène fut accompagnée par une absence de traduction. En 1994, la revue « LOUTKÁ̌̆ » publia la traduction de la pièce pour 
marionnettes, Duvelor ou la Farce du diable vieux, traduite par Simona Pěničková32 ${ }^{2}$, ce qui ranima l'intérêt envers Ghelderode éteint depuis la mort de Konůpek en 1968. Pěničková, alors étudiante au département de marionnettes de la DAMU, mit cette œuvre en scène comme travail de fin d'étude et choisit cette pièce comme sujet de mémoire. En 1993, l'étudiante avait déjà présenté Sire Halewyn dans l'espace authentique de l'église du monastère Emmaüs alors non réhabilité. En 1996, soit trente et un ans après le recueil de Konůpek, de nouvelles traductions de la plume de Marie Stretti (en collaboration avec le metteur en scène Hubert Krejčí) parurent enfin. Il s'agit de trois des textes les plus fondamentaux de Ghelderode : L'école des bouffons, Escurial et Sortie de l'acteur ${ }^{33}$. Afin que le nombre de traductions tchèques publiées soit complet, il faut encore ici ajouter le fascicule intitulé Pašijové hry(Les Pièces de la Passion,2002) ${ }^{34}$ qui comprennent les pièces Barabas et Mademoiselle Jaire.

Il existe aujourd'hui dix-sept traductions tchèques publiées des pièces de Ghelderode, soit environ un tiers des textes parus. Les œuvres phares sont accessibles au lecteur tchèque et quelques autres existent en traductions de travail non publiées. Par soucis d'intégralité, ajoutons qu'en 1998 parut un choix de textes en prose sous le titre les Statues ou le Jardin malade ${ }^{35}$.

aru dans la seconde moitié des années 1990, peutêtre similaire à celui de Konůpek en 1967, même s'il ne fut pas accompagné par des études de qualité, n'engendra pas de répercussion marquante sur la scène tchèque. De 1990 jusqu'à nos jours, on compte pourtant dix-sept mises en scène, ce qui évidemment n'est pas peu ni pour Ghelderode ni pour le théâtre tchèque, mais en regardant ce nombre de plus près, on ne remarque pas non plus un grand engouement. La pièce la plus attractive pour les dramaturges est Magie Rouge montée trois fois d'après l'esthétique de Ben Jonson. Puis suivent Escurial, œuvre très appréciée par les acteurs, l'attrayant Sire Halewyn et étonnamment Christophe Colomb. Ces trois pièces connurent chacune deux mises en scènes. Ce retentissement minimal et cette trace quasi nulle dans la conscience théâtrale indiquent que Ghelderode reste aujourd'hui plutôt une curiosité dramaturgique et que son interprétation scénique reste superficielle. Il est pourtant encore nécessaire de mentionner que Ghelderode est un auteur fétiche dans les théâtres estudiantins. Depuis les années 1960, on y recense un tiers de la totalité des mises en scène (8). A une exception près, il n'existe pas de renseignements sur le fait que des troupes de théâtre amateur, qui jouissent d'une position spécifique au sein de la culture tchèque et qui approchent souvent des auteurs intéressants, n'aient monté une pièce de Ghelderode.

Parmi les mises en scène des pièces de Ghelderode montées en République tchèque ces vingt dernières années, un spectacle créé à Brno se révéla tel le fameux diamant noir. Le metteur en scène slovaque Peter Scherhaufer était, avec Tálska que nous avons déjà citée, le fondateur et le principal metteur en scène du Divadlo Husa na provázku ${ }^{36}$, où, de 1967 à sa mort en 1999, il produisit plusieurs mises en scène essentielles. Le spectacle L'École des bouffons $s^{37}$ fut malheureusement sa dernière création; quelques jours après la première, le metteur en scène, sérieusement malade, mourut. Le choix de ce texte par lequel maître Folial dit adieu à ses disciples, Ghelderode à l'écriture théâtrale et Scherhaufer à la vie, peut effarer par sa fatalité. L'intrigue prenait place sur une scène simple surmontée de quelques marches, l'espace du couvent gothique et sombre imaginé par Ghelderode était retranché. Et, tout au long de la pièce, le metteur en scène s'en tirait finalement par des moyens simples, sans prétentions et expressifs. Le déplacement, dans la conception du chœur, des apprentis bouffons auquel prenait part presque toute la troupe du théâtre 
constituait un changement marquant. Aux orques terrifiants de Ghelderode, Scherhaufer mêla d'autres types de bouffons (il y avait même des femmes et des enfants), en particulier des clowns de cirque, ce qui donnait plus à la mise en scène un ton compatissant que celui d'une barbarie fatale. La notion de barbarie mélangée à la fugacité de la vie humaine pouvait ici acquérir une nouvelle teneur. Dans la conception de Scherhaufer, il ne s'agissait pas de la grimace gothique d'une époque vide, mais plutôt de la cruauté arrangée par une poétique plus subtile (le clown), qui n'utilisait pas de références affichées d'une mauvaise compréhension d'Artaud. Dans le rôle du maître Folial excellait l'acteur Pavel Zatloukal, dont la métamorphose de vieillard malade en roi des bouffons comptait parmi les grandes expériences théâtrales. Le testament de ce grand metteur en scène fut reçu positivement par la critique théâtrale, et la version radiophonique qui s'est efforcée de respecter l'atmosphère de l'exécution théâtrale démontre surtout la singularité de cette mise en scène. En 2002, le célèbre metteur en scène Jan Amos Pitínský l'a enregistrée dans une distribution identique à celle du divadlo Husa na provázku pour les ondes de Český rozhlas.

Étant donné que nous avons quasi vingt pièces de Ghelderode traduites en tchèque et que nous avons recensé jusqu'à nos jours vingt six mises en scène sur treize titres, on peut considérer la situation de la réflexion scientifique sur cet auteur est au plus bas. L'unique source d'information était déjà évoquée à partir des années 1960 dans les textes de Konůpek et Černý. La plupart des textes plus détaillés dans les programmes des spectacles ou dans les petits encarts répètent à maintes reprises des faits biographiques rapportés (souvent avec des erreurs) et recourent de manière caractéristique au séduisant cliché de "l'auteur sombre». Seuls les travaux sporadiques des étudiants viennent volontiers troubler ce vide d'explication. L'auteur de la présente étude a tenté d'apporter un regard plus complexe lorsqu'en 2006, il a défendu au département des Sciences théâtrales à Prague un travail intitulé Chapitre sur les marionnettes, la marionnettisation, les mannequins et autres engeances chez Michel de Ghelderode (Kapitola o loutkách, loutkovitosti, manekýnech a jiné havěti u Michela de Ghelderode) ${ }^{38}$ qui, outre le phénomène décrit, a tâché surtout de récapituler les recherches existantes sur Ghelderode. Notre autre texte publié s'intéresse au phénomène des métamorphoses dans le récit des Passions à travers les pièces de Ghelderode ${ }^{39}$.

Comme nous l'avons vu, Ghelderode en République tchèque n'appartient pas aux auteurs qui sont joués dans les grands théâtres institutionnels. On remarque un intérêt plus grand envers cet auteur auprès des groupes expérimentaux et des théâtres d'étudiants, des troupes avec une dramaturgie ouverte qui vraisemblablement fascine par son art et sa plasticité théâtrale, par sa possibilité étendue d'interprétations et sa thématique de cruauté, tellement actuelle dans le monde du $\mathrm{XX}^{\mathrm{e}}$ siècle et celui d'aujourd'hui. Ces attributs ont constitués des pièges dramaturgiques et scéniques pour de nombreux metteurs en scène et souvent leurs interprétations ne justifient pas de réduire la suggestivité apparente de cette surface impressionnante. Néanmoins, il résulte de ce panorama succinct et mince de Ghelderode en République tchèque qu'il y a eu une certaine " ghelderodite aiguë» à la fin des années 1960, lorsque les textes de l'auteur inspirèrent puissamment des metteurs en scène et donnèrent une série de traduction et quelques études. Il ne faut vraisemblablement pas s'attendre à un intérêt futur considérable pour les pièces de cet auteur même si, ici et là, résonnent des voix de professionnels de la scène sur la qualité de certains titres. Il semble qu'il n'y ait ni de courage dramaturgique ni d'interprétation scénique suffisamment fondée de cet auteur 
de la cruauté. Ghelderode n'a jamais eu, même à l'échelle mondiale, son grand metteur en scène et ces mariages sont encore plus rares aujourd'hui. Pourtant de grandes pièces, telles Barabas ou Sortie de l'acteur,attendent encore et toujours d'être montées en République tchèque...

\section{NOTES}

1. Parvi J., "Ghelderode en Pologne » in: Michel de Ghelderode et le théâtre contemporain, Société Internationale des Études sur Michel de Ghelderode, Bruxelles, 1980, pp. 241-255. Rjevskaja N., «Ghelderode en Russie », Ibid., pp. 127-132. Szanto J., «Les premières représentations de Ghelderode en Hongrie » in : Michel de Ghelderode dramaturge et conteur. Bruxelles, 1983, pp. 79-85. Hauger G., « Ghelderode en Grande-Bretagne - hélas ! », in : Ibid., pp. 85-90. Stolarek Zb., « Vivre avec Ghelderode en Pologne », in : Ibid., pp. 91-97.

2. En français dans le texte (NdT). Beyen R., Bibliographie de Michel de Ghelderode, Palais des Académies, Bruxelles, 1987.

3. La Pologne ne peut résolument pas se plaindre du manque et de la qualité de ses mises en scène. Elle occupe l'une des places principales en ce qui concerne le nombre de mises en scène produites. Les Polonais montrent une préférence pour Magie rouge qui est la pièce de Michel de Ghelderode la plus montée, suivie par les classiques La Balade du Grand Macabre, L'École des bouffons , Escurial et Sortie de l'acteur. Voir : Stolarek Zb.., Art. cit.

4. Beyen R., Op. cit., p. XVIII.

5. L'existence du "Théâtre derrière la porte ", " dérangeant » pendant la normalisation, prit fin en 1972 suite à une intervention des autorités de la normalisation. Vingt huit ans plus tard, en 1990, à l'occasion de l'ouverture du « Théâtre derrière la porte II ", le metteur en scène Vít Vencl revint vers le texte de Ghelderode Masques ostendais, et produisit une sorte de reconstitution de la mise en scène d'Otomar Krejča.

6. Krejča mentionne sa mise en scène dans son texte «Le regard du masque », in : Le Masque: Du rite au théâtre, textes réunis et présentés par Odette Aslan et Denis Bablet, Éditions du Centre National de la Recherche Scientifique, Paris, 1985, pp. 203-208.

7. Pour plus de renseignements sur la tournée belge, voir Beyen R., Op. cit., p. 463-464.

8. En fait, il s'agissait de la première présentation d'Antonin Artaud en Tchécoslovaquie.

9. Tři herci, jedno drama (Trois acteurs, un drame...), traduit du français par Jiří Konůpek, in : « $\mathrm{S}$ VĚTOVÁ LITERATURA ", 1966, nº 11/5, pp. 173-178.

10. Slepci : Moralita o jednom dějství, (Les Aveugles. Pièce en un acte), traduit du français par Jiř́i Konůpek, in : Ibid., pp. 168-172.

11. Kryštof Kolumbus, in :«DIVADLO », 1966, n 17/3, pp. 23-31.

12. Balada o velkém kostlivci, Dilia, Prague, 1966, pp. 152.

13. Pantagleize - Straka na šibenici, Dilia, Prague, 1967, p. 219.

14. Rudá magie a jiné hry. Orbis, Prague, 1967, p. 229.

15. Konůpek J., Tajůplný Michel de Ghelderode, in : « SVĚTOVÁ LITERATURA », 1966, nº 11/3, pp. 158167.

16. Konůpek J., «Dramatické dílo Michela de Ghelderode ", in : Rudá magie a jiné hry, Orbis, Prague, 1967, pp. 217-230.

17. Lepage A., Michel de Ghelderode, Dutilleul, Paris, 1960, p. 62. 
18. Černý J., "Ghelderodův flanderský sen » (Le rêve flamand de Ghelderode), in : « DIVADLO », $1968, n^{\circ} 19 / 1$, p. 48.

19. Beyen R., Op. cit., p. XXVIII.

20. Černý J., Art. cit., p. 43.

21. Sire Halewyn (Sir Halewyn), Divadlo Husa na provázku, Brno, première le 24 mars 1969, mise en scène de E. Tálská.

22. Tři herci, jedno drama, JAMU Brno-činoherní studio, première le 14 octobre 1969, mise en scène de J. Růžička.

23. Camus A., Le Malentedu (en tchèque: Nedorozuměni). Vedené divadlo, Prague, première le 22 décembre 1969, mise en scène de K. Makonj.

24. Hop, Signore! (en tchèque : Hop, Signor!), Vedené divadlo, Prague, première le 17 mai 1970, mise en scène de K. Makonj.

25. Preisner R. Exkurs o loutce a loutkovitosti (Digressions sur la marionette et la marionettisation)., in : «LOUTKÁ̌̌ », 2003, n 53/6, pp. 1-4. Complément séparé de la revue « LOUTKÁ̌̌ »; réimprimé de: Preisner R. , Johann Nepomuk Nestroy : tvưrce tragické frašky (Johann Nepomuk Nestroy, créateur de pièce tragique), Orbis, Prague, 1968. p. 265.

26. Ravon G., , « En courant. Vent du Nord », in : «LE FIGARO », 20 février 1950. Cité par Beyen R., Michel de Ghelderode ou la Hantise du masque: Essai de biographie critique, Palais des Académies, Bruxelles, 1971, p. 325.

27. Les Aveugles (Slepci), Loutkové divadlo Radost, Brno, première le 26 mars 1971, mise en scène de P. Vašíček.

28. La Balade du Grand macabre(en tchèque: Balada o velkém kostlivci), Naivní divadlo, Studio Ypsilon, Liberec, 14 juin 1973, mise en scène de J. Schmid.

29. Le « Divadelní pouŤ » se tint chaque été de 1985 à 1990 à Prague sur l'île Střelecký.

30. $O$ Ďáblovi, který sliboval hory doly, Studio Ypsilon, Prague, 16 sep-tembre 1986, mise en scène de J. Schmid. (Première le 18 juin 1986).

31. Sur le thème des « Passions » chez Ghelderode, voir les textes suivants : Petruželka H., « Actus groteskní - poznámky k podobám pašijového př́iběhu u Michela de Ghelderode ", in : " DIVADELNí REVUE ", 2008, n¹9/2, pp. 31-36. Hyvnar J., Dvojí pojetí pašijí u Michela de Ghelderode (La double conception de la Passion chez Michel de Ghelderode), 2004, p. 5. Texte non publié, dans les archives de l'auteur.

32. Duvelor ou la Farce du diable vieux, d'apès les vieux textes pour marionnettes rédigés par Michel de Ghelderode en 1931. Traduit du français par Simona Pěničková avec l'aide de Josef Balvín, in : « LOUTKÁŘ », 1994, n 44/55, p. 112-113.

33. deGhelderode M., Hry (Pièces), traduit du français par Mario Stretti, Divus, Prague, 1996, $134 \mathrm{p}$.

34. deGhelderode M., Pašijové hry (Les Pièces de la passion), traduit du français par Tomáš Kybal et Luděk Kárl, Dauphin, Prague, 2002, 201 p.

35. de Ghelderode M., Sochy aneb Nemocná zahrada (Les statues ou le jardin malade), traduit du Anna Kareninová, Zdeněk Hrbata et Tomáš Kybal, ANNO, Prague, 1998, 213 p.

36. Zdeněk Pospíšil était également l'un des fondateurs du théâtre.

37. Škola šaškủ (L'Ecole des bouffons), Husa na provázku, Brno, 24 avril 1999, mise en scène de P. Scherhaufer.

38. Petružela H., « Kapitola o loutkách, loutkovitosti, manekýnech a jiné havěti u Michela de Ghelderode » (Chapitre sur les marionnettes, la marionnettisation, les mannequins et autres engeances chez Michel de Ghelderode), Mémoire de maîtrise, Département des Sciences théâtrales, Faculté des Lettres, Université Charles de Prague, 2006, 118 p.

39. PetruželaH., « Actus groteskní...», Art. cit., pp. 31-36. 
INDEX

Index géographique : Belgique, République tchèque, Tchécoslovaquie Index chronologique : communisme, entre-deux-guerres, XXe siècle Mots-clés : littérature belge, littérature tchèque

\section{AUTEURS}

\section{HONZA PETRUŽELA}

Doctorant en Études théâtrales à l'Université Charles de Prague 